\title{
EDITORIAL
}

\section{Advancing Partnered Research in the VA Healthcare System: The Pursuit of Increased Research Engagement, Responsiveness, and Impact}

\author{
Martin P Charns, DBA, MBA ${ }^{7,2}$, Leonard E Egede, MD, MS 3,4 , John S Rumsfeld, MD, PhD ${ }^{5,6}$, \\ Geraldine C McGlynn, MEd7, and Elizabeth M. Yano, PhD, MSPH ${ }^{8,9}$
}

${ }^{1}$ Center for Healthcare Organization and Implementation Research (CHOIR), VA Boston Healthcare System, Boston, MA, USA; ${ }^{2}$ Department of Health Policy \& Management, School of Public Health, Boston University, Boston, MA, USA; ${ }^{3}$ Health Equity and Rural Outreach Innovation Center (HEROIC), Charleston VA HSR\&D Center of Innovation, Charleston, SC, USA; ${ }^{4}$ Center for Health Disparities Research, Medical University of South Carolina, Charleston, SC, USA; ${ }^{N}$ National Program Director for Cardiology, Veterans Health Administration, Washington, DC, USA; ${ }^{6}$ Division of Cardiology, School of Medicine, University of Colorado, Denver, CO, USA; ${ }^{7} \mathrm{HSR} \& D$ Center for Information Dissemination and Education Resources (CIDER), VA Boston Healthcare System, Boston, USA; ${ }^{8}$ VA HSR\&D Center for the Study of Healthcare Innovation, Implementation and Policy, VA Greater Los Angeles Healthcare System, Sepulveda, CA, USA; ${ }^{9}$ Department of Health Policy \& Management, UCLA Fielding School of Public Health, Los Angeles, CA, USA.

J Gen Intern Med 29(Suppl 4):S811-3

DOI: $10.1007 / \mathrm{s} 11606-014-3060-1$

(c) Society of General Internal Medicine 2014

$\mathrm{O}$ rganization scholars and astute leaders have known for half a century that involving people in decision-making increases their motivation to implement decisions ${ }^{1}$ and that integrating research and practice in an organization is associated with higher organizational performance. ${ }^{2}$ Yet this knowledge has not been used very effectively in implementing research evidence into practice, and the pipeline metaphor of translating research into practice inherently ignores these organizational facts. The pipeline portrays the development of knowledge as the domain of researchers, to be translated into usable form for practitioners and organizations. It also portrays a sequential process that leaves the potential users of research out of its development, contrary to what is needed to motivate the use of research findings. Conducting partnered research and evaluation through bidirectional communication and engagement of key stakeholders holds significant promise for improving the impact of research through the design and implementation of evidence-based practice and policy.

\section{WHAT IS PARTNERED RESEARCH?}

Ovretveit et al., in a review of the literature, augmented by interviews and discussions with colleagues in both research and practice as well as reflections on their own experience, proposed five criteria for characterizing research-practice partnerships ${ }^{3}$ :

- "researchers contribute to actions taken by practitioners or community members acting on or at one or more levels of a health system;
- studies are intended to produce quickly and directly actionable findings, but also to be published in peerreviewed scientific journals;

- studies involve both researchers and practitioners in defining the research questions and interpreting the findings;

- studies involve a 'significant' amount of time or other contributions (such as a data gathering system) from both researchers and practitioners; and

- a formulated description of the partnership approach has been published, with aims and methods, and there are published empirical studies showing the approach as applied (i.e., not just a conceptual description of a possible approach without examples)."

Attributes of partnered research represented in the articles in this supplement vary significantly in terms of the type of partners (i.e., number of partners and partnership levels), the genesis of the partnership and its goals, and the level of partner engagement.

Type of Partners. The majority of the papers in the supplement describe partnerships with program offices in the Veterans Health Administration (VHA) in Washington DC. The VHA is organized into policy and operations offices that oversee all clinical programs nationwide. Research partnerships at this level of the organization have a greater opportunity to influence national policy and practice directives and to inform evidence-based strategic planning. Other papers focused on more field-based partnerships - for example, with one or more of the 21 Veterans Integrated Service Networks (VISNs) and/or local VA medical centers (VAMCs) - closer to the point of care and delivery of routine practice. Most papers also represented two or three levels, likely as a result of the complexity and difficulty inherent in managing increasingly 
diverse webs of stakeholders. Very few papers represented a range of partnership levels recommended for improving research impacts on practice or population health (i.e., patient/ family, provider, practice, community, state, and national health policy), but that is true of the larger body of literature as well. ${ }^{4,5}$ As a national integrated healthcare delivery system, VA partnered research adds potential levels for consideration, including researchers, clinic managers, department chairs, service line leaders, VAMC directors, VISN leaders, and VHA program leaders. However, we also lack broad standards for reporting on partnered research. Hysong and colleagues seek to advance such standards for publishing partnered protocols.

Genesis of the Partnerships. These papers demonstrate both top-down and bottom-up initiatives to develop partnerships capable of generating, implementing, and disseminating evidence-based practice. Top-down initiatives often represented exigencies emanating from critical policy issues where policymakers and operations leaders recognized the value of engaging researchers in critical appraisal of the determinants of an issue and/or systematic evaluation of new programs or care models. Wu and colleagues' lung cancer screening (LCS) program is an example of a top-down initiative. Senior VHA program leaders tasked the National Center for Health Promotion and Disease Prevention (NCP) with developing an LCS program. NCP, in turn, reached out to systems engineers to develop needed electronic clinical tools, and to health services researchers to lead the program evaluation. The multilevel partnership among policy, clinical, and research leaders aimed to inform potential national system-wide implementation, depending on evaluation results. Kertesz and colleagues evaluated implementation of the VA's Housing First program and benefitted from major top-down initiatives to reduce, if not eliminate, homelessness by 2015 , resulting in significant funds to tackle the multidimensional issues driving veteran homelessness. This partnership, however, is described as a more "arm's-length" relationship, emphasizing the independent interests of the investigative group from those of the VA National Center on Homelessness Among Veterans. The National Center provided early guidance to refine the researchers' study question, convened two expert panels, and reviewed and discussed study findings in interim reports and draft and final manuscripts. This two-level partnership reflects a more traditional dissemination model, with distinct roles established in order to minimize potential conflicts of interest in the interpretation and reporting of results. Damush and colleagues describe another two-level partnership between the VHA Offices of Emergency Medicine and Specialty Care Services and the Stroke Quality Enhancement Research Initiative (QUERI) Center researchers to evaluate implementation of acute stroke care centers. The QUERI team evaluated implementation barriers and facilitators and presented results to the operational partners, and then added a level of dissemination by establishing monthly virtual meetings with field-based stroke clinicians to increase engagement.
Bottom-up initiatives, in contrast, typically represented researcher-initiated efforts to collaborate with VA leaders to ensure that their research was adapted to the practical, contextual realities of the healthcare system and/or to engender stakeholder engagement to increase the likelihood of implementation, proliferation, and sustainability of research evidence into routine practice and policy. An example of bottom-up partner engagement is reflected in Dobscha's initiation of a dialogue with the VA Mental Health Services' Suicide Prevention Office in an effort to identify opportunities to intervene with veterans seen in primary care settings who may be at risk of suicide. They then developed a data-sharing agreement with a VISN suicide prevention center to examine a decade's worth of state death certificates, linking them with VA utilization data to characterize patterns of primary care use among veterans who had committed suicide. Results of this three-level partnership helped to inform VA mental health strategic planning. Zulman's paper is another bottom-up example where field-based researchers worked with VAMC leadership and clinicians to redesign processes in order to improve primary care for high-need, high-cost patients. While this was also a three-level partnership, albeit at lower tiers of the system, the level of local engagement was much greater than in the Dobscha example, reflecting more of a multilevel dissemination effort. Zulman's work is also having direct influence on national strategic planning as part of a national demonstration project. The work by Dorflinger and colleagues in planning, implementing, and analyzing the process of adoption of a pain management and opioid therapy program is another bottom-up example. They developed an interdisciplinary clinical research team with VAMC-level quality managers, educators, and clinicians. The goal of this three-level local partnership was to sustain meaningful engagement of the stakeholders who would "own" the program moving forward.

Damschroder describes a two-level partnership between researchers and the VHA office responsible for monitoring performance that is neither top-down nor bottom-up. Essentially, the Office of Informatics and Analytics capitalizes on the technical expertise of researchers to develop and test new clinical action measures and to evaluate the properties of existing performance measures in direct collaboration and ahead of national implementation. As VA focus on partnered research increases, we anticipate more direct collaborations like this one in key topical areas of research expertise.

The supplement contains one example of a multi-agency partnership and another example of a public-private partnership. Nieuwsma describes a 38-member multidisciplinary group of representatives from mental health and chaplaincy services in the Departments of Veterans Affairs and Defense. Their goals were to effectively engage stakeholders and subject matter experts in the design, data collection, analysis, interpretation, and application of findings from a mixedmethods study of the integration of mental health and chaplain services within and across agencies. Similar to other multilevel partnerships, this effort was designed to ultimately increase the 
speed of translation of study findings into actionable recommendations. Elwy describes efforts to improve large-scale adverse event disclosures through partnerships with VA leaders and policymakers within and outside healthcare, as well as with private sector consulting groups working with the VA on these issues. Partnered activities were focused on collaborative identification of critical disclosure processes and dissemination of jointly developed recommendations to the field, chiefly through presentations and a white paper.

Levels of Engagement. Two papers demonstrated higher levels of partner engagement. Midboe et al. describe explicit use of community-based participatory research models in their highly collaborative partnership with the Office of Clinical Public Health, which oversees HIV testing within the VA. They describe lessons learned about respecting different time horizons among partners, identifying relevant research questions for both parties, designing flexible studies, and engaging partners at each stage of research. Kirchner and colleagues describe the outcomes of a multilevel partnered facilitation strategy for implementing primary care-mental health integration (PC-MHI). In this model, researchers served as consultants, providing implementation science expertise, tools, and resources needed to support implementation. VA regional and facility leadership provided expertise in their organizational structures and clinical processes, and identified typical implementation barriers and facilitators within their network. Internal facilitators, in turn, partnered with providers and regional, facility, and clinic managers to implement PC-MHI.

We hope that the articles in this supplement provide useful insights into the range of opportunities that partnered research represents for advancing the implementation and proliferation of research evidence into routine practice. We posit that increasing the types of partners, building on multilevel topdown and bottom-up collaborations, and increasing the nature of stakeholder engagement in the design, conduct, and application of research findings will establish a new roadmap for rapid implementation. ${ }^{6}$

Acknowledgements: The supplement described in this editorial was funded by the VA Health Services Research and Development (HSR\&D) Service in the Office of Research and Development, Veterans Health Administration. Dr. Yano's effort was funded by a Senior Research Career Scientist award (Project \# RCS 05-195).

Corresponding Author: Martin P Charns, DBA, MBA; Center for Healthcare Organization and Implementation Research (CHOIR)VA Boston Healthcare System, 150 S. Huntington Avenue, Boston, MA, USA (e-mail: martin.charns@va.gov).

\section{REFERENCES}

1. Vroom VH, Yetton PW. Leadership and decision-making. Pittsburgh: University of Pittsburgh Press; 1973.

2. Lawrence PR, Lorsch JW. Differentiation and integration in complex organizations. Admin Sci Quarterly. 1967;12:1-47.

3. Ovretveit J, Hempel S, Magnabosco JL, Mittman BS, Rubenstein LV, Ganz DA. Guidance for research-practice partnerships (R-PPs) and collaborative research. J Health Org Manage. 2014;28(1):115-126.

4. Yano EM, Green LW, Glanz K, Ayanian JZ, Mittman BS, Chollette V, Rubenstein LV. Implementation and spread of interventions into the multilevel context of routine practice and policy: Implications for the cancer care continuum. J Natl Cancer Inst Monogr. 2012;44:86-99.

5. Taplin SH, Rodgers AB. Toward improving the quality of cancer care: Addressing the interfaces of primary and oncology-related subspecialty care. J Natl Cancer Inst. 2010;40:3-10.

6. Kessler R, Glasgow RE. A proposal to speed translation of healthcare research into practice: Dramatic change is needed. Am J Prev Med. 2011;40(6):637-644. 